\title{
Current-induced vortex trapping in asymmetric toothed channels
}

\author{
J. Cuppens, G. W. Ataklti, and V. V. Moshchalkov \\ Institute for Nanoscale Physics and Chemistry, K.U. Leuven, Celestijnenlaan 200D, B-3001 Leuven, Belgium
}

A. V. Silhanek

Institute for Nanoscale Physics and Chemistry, K.U. Leuven, Celestijnenlaan 200D, B-3001 Leuven, Belgium and Département de Physique, Université de Liège, B-4000 Liège, Belgium

J. Van de Vondel

Institute for Nanoscale Physics and Chemistry, K.U. Leuven, Celestijnenlaan 200D, B-3001 Leuven, Belgium and Institut Catalá de Nanotecnologia (ICN), Centre d'Investigació en Nanociència i Nanotecnologia (CIN2), Campus Universitat Autònoma de Barcelona, Bellaterra, Barcelona E-08913, Spain

C. C. de Souza Silva, R. M. da Silva, and J. Albino Aguiar

Departamento de Física, Universidade Federal de Pernambuco, Cidade Universitária, 50670-901 Recife-PE, Brazil

(Received 4 August 2011; published 8 November 2011)

\begin{abstract}
We investigate experimentally and theoretically the vortex dynamics in a superconducting film with nanoengineered open vortex traps which provide tunable pinning, ranging from no pinning for a certain current direction to finite pinning when reversing the current flow. The design is based on the confinement of the vortex motion within two repulsive walls, one with periodic microprotrusions and the other one smooth. Clear commensurability effects are seen if the bias current drives the vortices inside the traps, whereas these effects are much less pronounced when the current pushes the vortices against the smooth surface. For small periods of the protrusions or large vortex sizes, the properties displayed by the vortices, pushed against these two surfaces of dissimilar roughness can be thought of as due to the change of effective sliding friction.
\end{abstract}

DOI: 10.1103/PhysRevB.84.184507

PACS number(s): 74.78.-w, 74.25.Wx

\section{INTRODUCTION}

During the past decades we have witnessed a sustained effort to improve the vortex pinning properties of type-II superconductors. ${ }^{1}$ Irrespective of the nature of the vortex pinning, i.e., core, electromagnetic, or stress induced, once a vortex is trapped, it needs extra energy to escape from the potential well, the so-called pinning energy $U_{p}$. A current flowing in the superconductor can provide this extra energy and induce the vortex depinning when $J \Phi_{0} d \gtrsim U_{p} / r_{p}$, where $J$ is the electric current density, $\Phi_{0}$ is the flux quantum unit, $r_{p}$ is the characteristic pinning range, and $d$ is the film thickness. ${ }^{2}$ In other words, the higher the current the smaller the influence of the pinning potential. ${ }^{3}$

In this work we propose, design, and investigate an artificial pinning landscape based on repulsive pinning centers, ${ }^{4-7}$ which increases its pinning efficiency if a current is applied in certain directions. We rely on a so far unexplored mechanism to control the flow of vortices by changing their effective sliding friction against potential walls of contrasting rugosity. We study a two-dimensional pattern as schematically shown in Fig. 1(a), where vortices are channeled between a smooth repulsive wall and a rough surface with small protrusions. An external force parallel to the channels will force the flux to flow along them, whereas a force perpendicular to the channels can push the vortices into the grooves [Fig. 1(c)] and consequently decrease their mobility or even completely suppress their motion. A perpendicular force in the opposite direction will direct the vortices against the smooth wall, allowing them to move unhindered [Fig. 1(b)].

\section{SAMPLE DETAILS}

To check these ideas, we performed transport measurements on a superconductor-superconductor hybrid system comprising a 50-nm-thick patterned $\mathrm{Pb}$ layer deposited on top of a 40-nm-thick Al film . To minimize proximity effects, we separate the $\mathrm{Al}$ film from the $\mathrm{Pb}$ film with a $10 \mathrm{~nm}$ insulating Ge layer. We investigate two coevaporated samples with different $\mathrm{Pb}$ patterns: (i) a sample with a periodic array of stripes with protrusions $(a=3.5 \mu \mathrm{m})$ where the teeth length and width are $l=1 \mu \mathrm{m}$, and the teeth separation is $b=1.5 \mu \mathrm{m}$ [Fig. 1(a)]; and (ii) a reference sample comprising long parallel stripes without protrusions, $1 \mu \mathrm{m}$ wide and also with a period of $3.5 \mu \mathrm{m}$.

In both samples the stripes are aligned at $45^{\circ}$ relative to the transport current direction. Hence, the induced Lorentz force components parallel $\left(F_{x}\right)$ and perpendicular $\left(F_{y}\right)$ to the $\mathrm{Pb}$ stripes are the same. By inverting the current polarity (here, positive current means $F_{x}<0$ and $F_{y}>0$ ), it is possible to flip both $F_{x}$ and $F_{y}$. For the sample without protrusions, because of the reflection symmetry along both $x$ and $y$ directions, changing force signs should have no effect on the vortex dynamics. This is not the case for the sample with protrusions: a negative (positive) force $F_{x}$ is expected to push vortices into (away from) the grooves, thus allowing vortices to probe different kinetic frictions. 


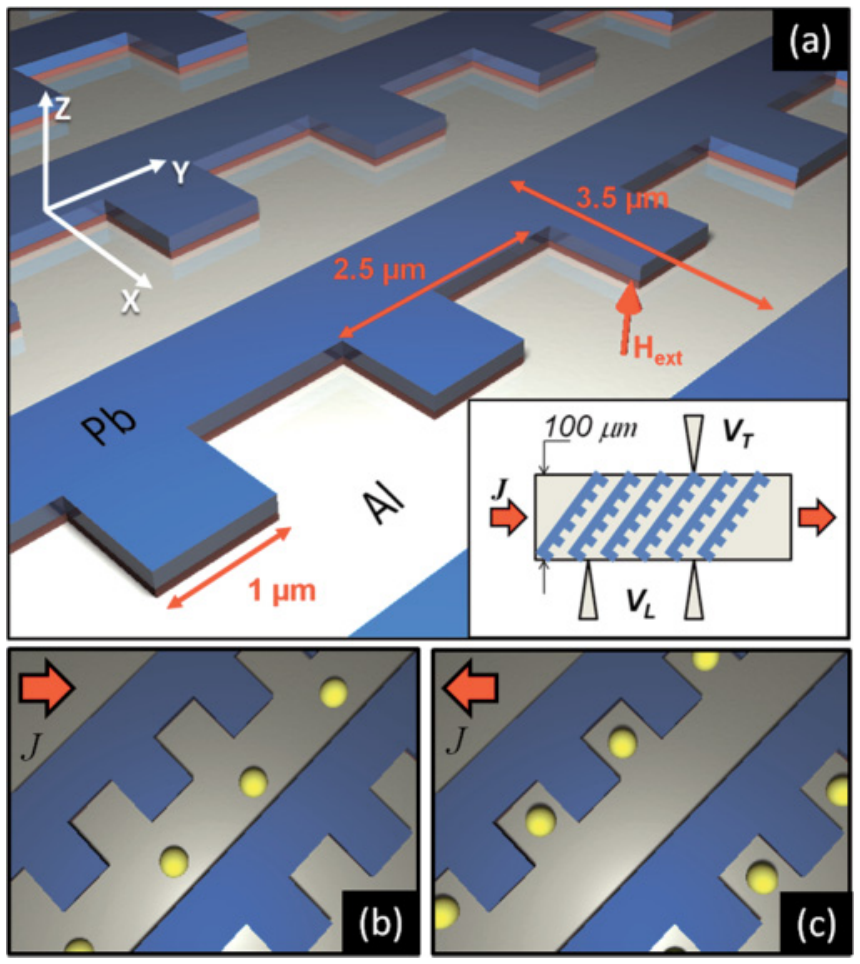

FIG. 1. (Color online) (a) Schematic representation of the sample layout. The comb-shaped $\mathrm{Pb}$ stripes guide the vortices in the $\mathrm{Al}$ superconducting film underneath. The current in the Al bridge flows in the $x-y$ plane at $45^{\circ}$ relative to the $x$ axis. The inset of panel (a) is a zoom out indicating the contacts' configuration. In (b) and (c) the yellow dots indicate the vortices for a positive field in the Al bridge for current flowing from left to right (b) and from right to left (c).

\section{EXPERIMENTAL RESULTS}

The first step consists of demonstrating that the $\mathrm{Pb}$ stripes indeed act as repulsive walls. To that end, the sample was zero-field cooled down to a temperature just above the transition temperature of the Al layer $\left(T_{c 0}=1.323 \mathrm{~K}\right)$. Then a perpendicular magnetic field was ramped up to a given value, $H_{\text {exc }}$, and set back to zero. After that, the superconducting transition of the $\mathrm{Al}$ bridge is measured. By adopting a $50 \%$ normal state resistance criterion, the transition temperature for different $H_{\mathrm{exc}}$ is determined as shown in the inset of Fig. 2. For $H_{\mathrm{exc}}<13 \mathrm{mT}$ we found that the transition temperature is not affected by previous magnetic history, thus indicating that the $\mathrm{Pb}$ microstructures are in the Meissner state (i.e., they are not able to trap any magnetic flux). For $H_{\text {exc }}>13 \mathrm{mT}$, the $\mathrm{Pb}$ microstructures do trap flux, thus decreasing $T_{c}(H)$ of the Al film. Hereafter, all measurements have been performed at fields $|H| \leqslant 2 \mathrm{mT}$, ensuring no vortex trapping in the $\mathrm{Pb}$ stripes and a purely repulsive interaction between the vortices in the $\mathrm{Al}$ layer and the $\mathrm{Pb}$ stripes.

In order to identify the direction of the vortex motion we recorded the voltage drop in the Al bridge parallel $\left(V_{L}\right)$ and perpendicular $\left(V_{T}\right)$ to the current direction as schematically shown in the inset of Fig. 1(a). From $V_{L}$ and $V_{T}$ we can calculate, by simple geometrical arguments, the voltage response generated by vortices moving parallel to the stripes, $V_{y}$, and

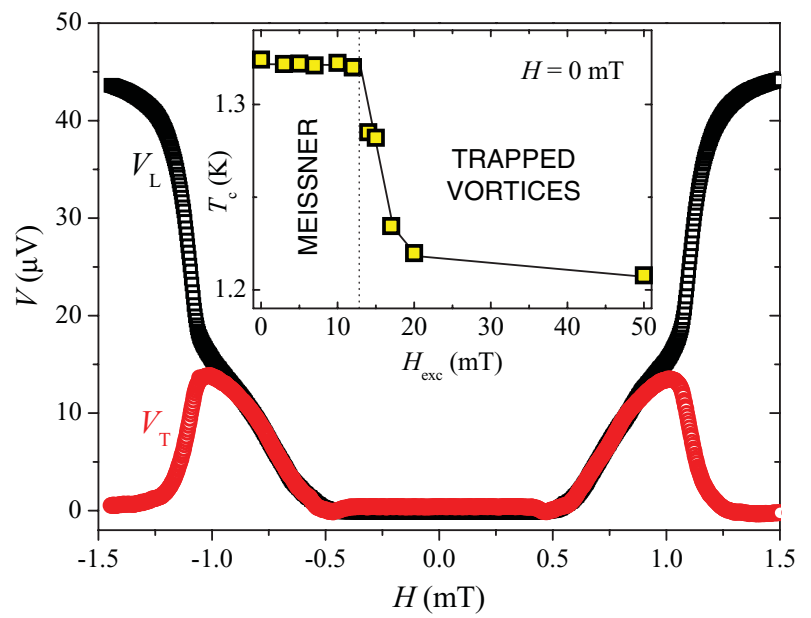

FIG. 2. (Color online) Voltage drop parallel $\left(V_{L}\right)$ and perpendicu$\operatorname{lar}\left(V_{T}\right)$ to the current direction. The vortices are perfectly channeled by the $\mathrm{Pb}$ stripes in the region where $V_{L}=V_{T} \neq 0$. The inset shows the critical temperature $T_{c}$ of the $\mathrm{Al}$ film at remanence $(H=0)$, after the field has been ramped up to a $H_{\text {exc }}$. The sudden reduction of $T_{c}$ at $\sim 13 \mathrm{mT}$ signals the onset field where magnetic moment is trapped in the $\mathrm{Pb}$ stripes.

perpendicular to them, $V_{x} \cdot{ }^{8}$ Note that here the subscripts $x$ and $y$ denote the direction of vortex motion and they are not to be confused with the actual direction of the induced electric field, which is perpendicular to the vortex motion. Since the separation between voltage probes is in both cases $100 \mu \mathrm{m}$, as long as the $\mathrm{Pb}$ stripes guide the vortex motion exactly at $45^{\circ}$ with respect to the current direction, we should obtain $V_{L}=V_{T}{ }^{9,11}$ This is indeed observed in Fig. 2 for the sample with protrusions at $T=1.231 \mathrm{~K}$ and $J=3 \mathrm{kA} / \mathrm{cm}^{2}$ for fields $|H| \leqslant 1 \mathrm{mT}$. Acquiring $V_{L}(H)$ and $V_{T}(H)$ simultaneously for different temperatures we can thus define the region where (i) vortices remain pinned $\left(V_{L}=V_{T}=0\right)$, (ii) the $\mathrm{Pb}$ stripes efficiently channel the vortex motion $\left(V_{L}=V_{T} \neq 0\right)$, and (iii) vortices in the $\mathrm{Al}$ bridge leak under the $\mathrm{Pb}$ stripes $\left(V_{L} \neq V_{T}\right)$. Figure 3 summarizes these measurements for both, the stripes with and without protrusions, for $J=3 \mathrm{kA} / \mathrm{cm}^{2}$. The onset of vortex motion is defined when $V_{L}+V_{T}>$ $5 \mu \mathrm{V}$, whereas the end of the guidance regime is given by $V_{L}-V_{T}>5 \mu \mathrm{V}$.

For the sake of simplicity now we will focus only on the guidance regime where the trajectory of the vortices is confined between two consecutive $\mathrm{Pb}$ stripes allowing us to reach a simpler interpretation of the measurements. Within this dynamic regime and for a fixed bias current direction along $-x$, we expect that vortices of positive polarity $(H>0)$ will be pushed against the protrusions and tend to be trapped in between them [Fig. 1(c)], whereas vortices of opposite polarity $(H<0)$ will slide with lower resistance when pushed against the smooth surface [Fig. 1(b)]. This predicted different dynamics between vortices of opposite polarity is confirmed in Fig. 4(a) by the $V_{y}$ measurements as a function of the normalized external magnetic field $H / H_{1}$, where $H_{1}=0.22 \mathrm{mT}$ is the field at which the density of vortices and traps coincide. In this figure it is clearly seen that for all explored fields, the dissipation, i.e., the vortex mobility, is always larger when the vortices are pushed against 


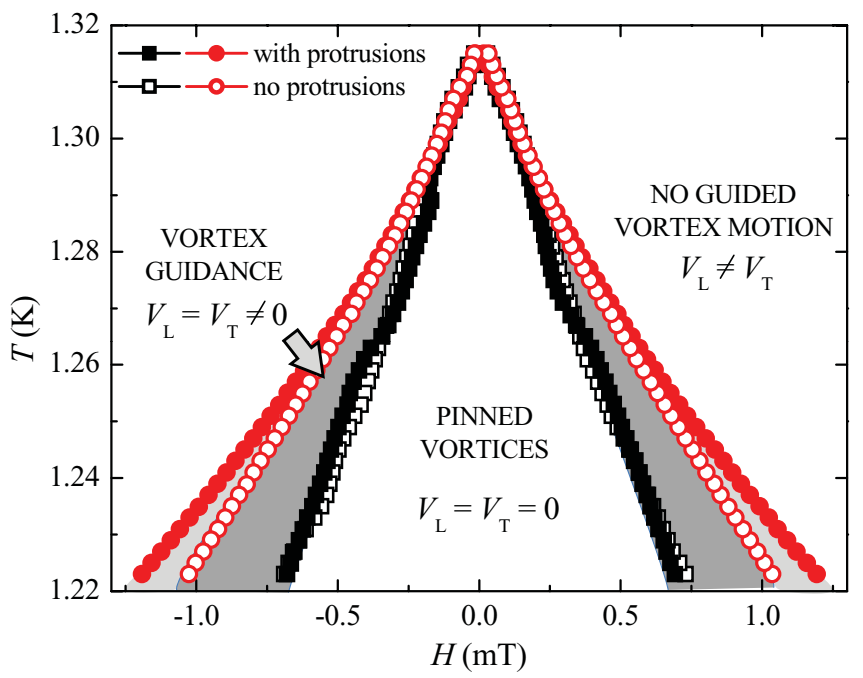

FIG. 3. (Color online) Temperature vs field dynamic phase boundary obtained from measurements similar to the one shown in Fig. 2 for samples with an array of combs and an array of $\mathrm{Pb}$ lines. The regime where perfect vortex guidance takes place has been shaded.

the smooth border. The signal $V_{y}(H)$ for the sample without protrusions is also shown here with a continuous line. As expected, no particular features are seen in this curve.

In general as long as the repulsive walls impede the motion of vortices along the $x$ direction, $V_{x}(H)=0$. In Fig. 4(a), for the sample with protrusions, we show that this scenario takes place for $H<H_{1}$, whereas for $H>H_{1}$ some vortices leak through the walls. Interestingly, approaching the field corresponding to two vortices per unit cell, $H=2 H_{1},\left|V_{x}\right|$

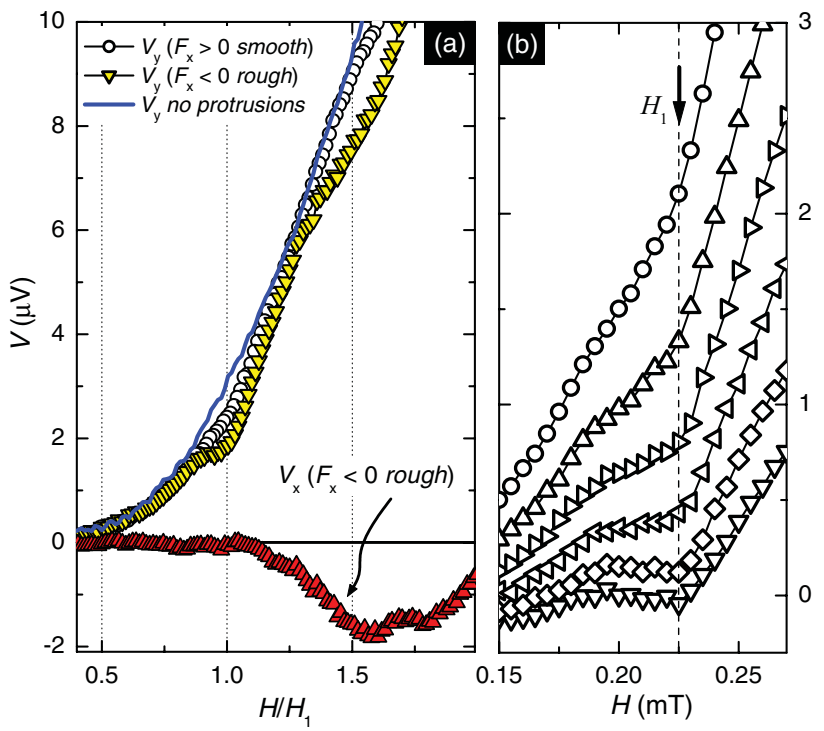

FIG. 4. (Color online) (a) $V_{y}$ as a function of the field for the sample with an array of combs for the two opposite driving forces $\left(F_{x}<0\right.$ and $\left.F_{x}>0\right)$ and for the sample with stripes $\left(F_{x}<0\right)$ (blue curve). These measurements were performed with $J=7.5 \mathrm{kA} / \mathrm{cm}^{2}$ and at $T=1.250 \mathrm{~K}$. (b) Evolution of the matching feature at $H=H_{1}$ for temperatures ranging from $T=1.260 \mathrm{~K}$ (top) to $T=1.235 \mathrm{~K}$ (bottom), in steps of $5 \mathrm{mK}$ with negative driving force. decreases even though the force against the repulsive wall has increased.

The most striking feature of Fig. 4(a) is that the asymmetry of the voltage response for the array of stripes with protrusions is particularly prominent at a field $H=H_{1}$. In other words, this so-called matching feature is more pronounced for positive fields than for negative fields. The fact that this feature remains located at the same field loci regardless of the temperature, as shown in Fig. 4(b), suggests that indeed it corresponds to geometrical commensurability effects. One of the most salient results of this paper is that the introduced open traps lead to matching features only if the current pushes the vortices inside the traps.

\section{THEORETICAL MODELING}

In order to illustrate how the geometry of our pattern is related to different friction mechanisms for different field polarities and to reveal the vortex trajectories corresponding to each phase in Fig. 4(a), we performed molecular dynamics simulations of the system discussed above. For that, we model the dynamics of $N$ vortices in a thin superconducting film by the equation of motion $\eta \vec{v}_{i}=\vec{F}-\sum_{j} \vec{\nabla}_{i} U_{i j}\left(r_{i j}\right)-$ $\vec{\nabla}_{i} U_{b}\left(r_{i}\right)$, where $\eta$ is the viscous drag coefficient, $\vec{F}$ is the Lorentz force induced by the transport current density, and $U_{i j}$ is the vortex-vortex pair potential, modeled as a Bessel interaction $U_{i j}=\epsilon K_{0}\left(r_{i j} / \lambda\right)$, where $\epsilon=\Phi_{0} /\left(4 \pi \mu_{0} \lambda\right)$ and $\lambda$ is the effective penetration depth given by $\lambda=\lambda_{L}^{2} / d\left(\lambda_{L}\right.$ is the London penetration length). The effect of the patterned layer

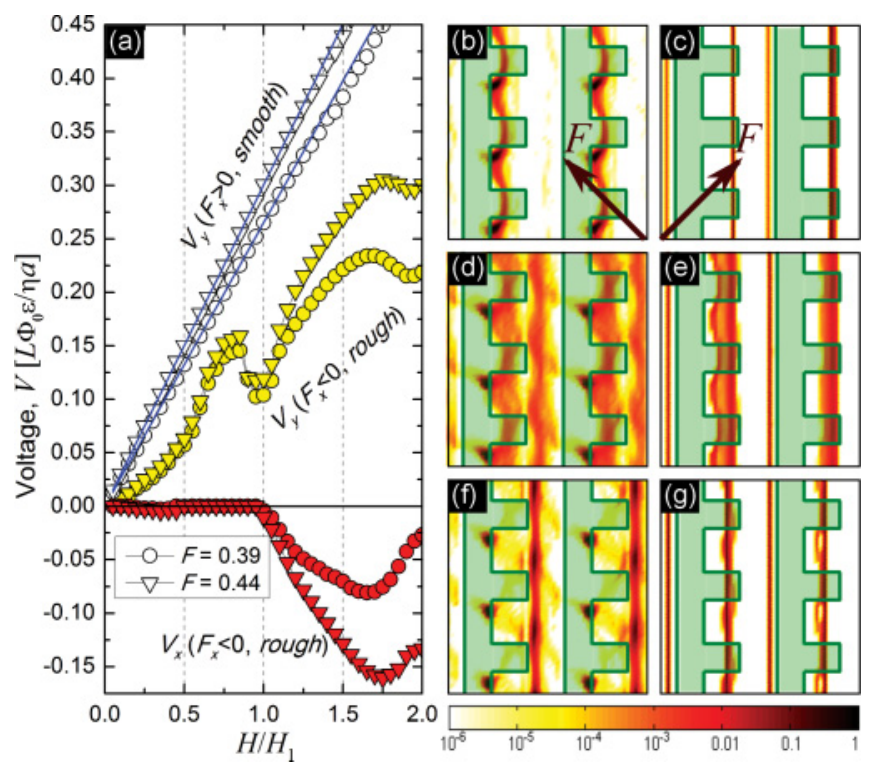

FIG. 5. (Color online) Simulation results. (a) Voltage drop induced by vortex motion along $x\left(V_{x}\right)$ and $y\left(V_{y}\right)$ for different orientations of $F_{x}$ and $F$ fixed at 0.39 and 0.44 for an array of stripes with protrusions. The solid blue lines show the corresponding data for an array of stripes without protrusions [(b)-(g)]. Density plots of the time-averaged vortex density $\rho(x, y)$ for $F=0.39$ and reduced fields $n=H / H_{1}=1.0$ [(b) and (c)], 1.5 [(d) and (e)], and 2.0 [(f) and $(\mathrm{g})]$, when the vortices are pushed toward the smooth side (right column) and to the rough side (left column). Only a small region $\left(7.0 \times 7.5 \mu \mathrm{m}^{2}\right)$ of the simulation cell is shown. 
is modeled in a simplified way by assuming that each area element of the pattern repels a vortex as a Gaussian barrier; that is, $U_{b}(r)=U_{0} \gamma \int_{\Sigma} d^{2} r^{\prime} \exp \left(-\left|\vec{r}-\vec{r}^{\prime}\right|^{2} / 2 \xi_{b}^{2}\right)$, where $\xi_{b}$ measures the range of the potential and $\gamma$ is a normalizing factor. The pattern area $\Sigma$ has the same geometry described in Fig. 1(a). We further assume $\lambda=2.5 a,{ }^{10} \xi_{b}=0.1 a$, and the barrier height $U_{0}=0.25 \epsilon$.

The calculations are performed on a $35 \times 35 \mu \mathrm{m}^{2}$ simulation cell (thus comprising $N_{g}=10 \times 14$ grooves) with periodic boundary conditions. For each vortex occupation number $n=N_{v} / N_{g}$, the system is equilibrated following a standard annealing scheme. Then, the value of the Lorentz force is swept while keeping its orientation fixed at either $\theta=$ $\pm 45^{\circ}$ with respect to the long bars ( $y$ axis), thereby allowing vortices to probe either smooth or rough sides of the channels. Figure 5(a) presents the voltage responses $V_{x}$ and $V_{y}$ induced by vortex motion along $x$ and $y$ directions, respectively $\left(V_{\alpha}=\right.$ $\Phi_{0} \sum v_{\alpha i} / L$, with $\alpha=x, y$ and $\left.L=35 \mu \mathrm{m}\right)$. The results are in good agreement with our experimental findings. When the vortices are pushed against the rough side of the channels, the $V(H)$ curves present clear matching features near $H_{1}$ and $2 H_{1}$, in contrast with the monotonic $V(H)$ curves for $\theta=45^{\circ}$. Moreover, vortices dissipate considerably less power when they are pushed against the rough surface as a result of its enhanced friction properties.

The roles of friction and matching features can be more clearly illustrated by calculating the time-averaged vortex distribution $\rho(x, y)$. Density plots of $\rho(x, y)$ are shown in Figs. 5(b)-5(g). For $\theta=45^{\circ}$, most vortices follow straightline paths due to the weak interaction with the patterned teeth. In contrast, for $\theta=-45^{\circ}$, vortices follow much more tortuous trajectories, trying to avoid the intersection point between the long bars and the teeth. In addition, their dynamics becomes very sensitive to the applied field. Particularly, for $n=1$, all vortices become temporarily trapped in the grooves before jumping to the next groove, which results in a slow slip-stick dynamics and the observed dark spots [that is, $\rho(x, y) \simeq 1$ ] near the grooves corners. For $n$ between 1.5 [Figs. 5(d) and 5(e)] and 2.0 [Figs. 5(f) and 5(g)], the slip-stick phase going on nearby the grooves coexists with the smooth motion of the excess vortices somewhat away from the grooves. However, a much more coherent motion occurs for $n=2.0$, as can be inferred from the filamentary profile of the excess vortex motion [Fig. 5(f)]. Such a spatial separation between this filamentary dynamics and the slip-stick motion is not as clear for nonmatching fields [Fig. 5(d)] and vortices participating on the different dynamical states interact strongly. This leads to a strong disturbance of vortices temporarily trapped in the grooves forcing them to leak through the groove corners toward a neighboring channel, thus resulting in a stronger response in the $x$ direction.

\section{CONCLUSION}

A similar concept of "dead ends" was used in the work of Wördenweber et al. ${ }^{12}$ in high $T_{c}$ superconducting thin films with a specific arrangement of antidots. In their work, the authors attributed the low current vortex propagation to a novel mechanism based on flux nucleation within the antidots due to the redistribution of screening currents and flux quantization. This mechanism differs from the conventional vortex motion observed in our devices. Also, it is worth noting that unlike in previous works by Pruymboom et al. ${ }^{13}$ and Kokubo et al. ${ }^{14}$ where vortices were confined by repulsive walls made from a strong pinning superconductor $(\mathrm{NbN})$ with trapped flux on top of a low pinning superconductor $\left(\mathrm{Nb}_{1-x} \mathrm{Ge}_{x}\right)$, our device is purely based on Meissner expulsion.

The particular choice of orientation of the $\mathrm{Pb}$ stripes on top of the Al film has been made merely as a way to illustrate the proof of principle of our device. The observed, currentdirection-dependent, vortex trapping could also be used to rectify vortex motion. ${ }^{12,15,16}$ In addition, more controllable systems can be obtained by combining two independent current sources along the $x$ and $y$ directions. ${ }^{17-19}$ In this case a vortex transistor can be developed where large voltage drops due to vortex motion along the $y$ direction can be controlled with small forces or gating in the $x$ direction. We expect the observed, current-direction-dependent, vortex-trapping mechanism can be magnified by making more repulsive cages with higher $T_{c}$ superconductors or magnetic templates with out-of-plane moment. Other measurements performed by us on different samples with the similar design, show the guidance can be greatly improved by making the Meissner stripes wider.

\section{ACKNOWLEDGMENTS}

This work was supported by Methusalem funding by the Flemish government, the Flemish Science Foundation, the Brazilian science agencies FACEPE/CNPq under Grant No. APQ-0589-1.05/08, CNPq under e Grant No. 309832/2007-1, and by the CNPq-FWO cooperation program. A.V.S. and J.V.d.V acknowledge support from FWO-Vl. J.C. thanks the Institute for the Promotion of Innovation through Science and Technology in Flanders (IWT-Vlaanderen). The authors are thankful to L. R. E. Cabral for useful discussions and the critical reading of the manuscript.

\footnotetext{
${ }^{1}$ Basic Research Needs for Superconductivity, Report on the Basic Energy Science Workshop on Superconductivity (2006).

${ }^{2}$ E. H. Brandt, Rep. Prog. Phys. 58, 1465 (1995).

${ }^{3}$ P. W. Anderson, Phys. Rev. Lett. 9, 309 (1962).

${ }^{4}$ E. H. Brandt, Phys. Status Solidi B 71, 277 (1975).

${ }^{5}$ W. Gillins, A. V. Silhanek, and V. V. Moshchalkov, Appl. Phys. Lett. 91, 202510 (2007).
}

${ }^{6}$ G. R. Berdiyorov, V. R. Misko, M. V. Milošević, W. Escoffier, I. V. Grigorieva, and F. M. Peeters, Phys. Rev. B 77, 024526 (2008).

${ }^{7}$ R. M. da Silva and C. C. de Souza Silva, Phys. Rev. B 83, 184514 (2011).

${ }^{8}$ If $\theta$ is the angle between the current direction and the stripes, $V_{y}=V_{L} \cos \theta+V_{T} \sin \theta$ and $V_{y}=-V_{L} \sin \theta+$ $V_{T} \cos \theta$. 
${ }^{9}$ N. Verellen, A. V. Silhanek, W. Gillijns, V. V. Moshchalkov, V. Metlushko, F. Gozzini, and B. Ilic, Appl. Phys. Lett. 93, 022507 (2008).

${ }^{10}$ Typical values for $\lambda(0)$ in an $\mathrm{Al}$ film are around $130 \mathrm{~nm}$, for a thickness $d=40 \mathrm{~nm}$ and reduced temperature $T / T_{c}=$ $1.25 / 1.323$, we find the effective penetration depth $\lambda=\lambda_{L}^{2} / d(1-$ $t)=7657 \mathrm{~nm} \sim 2.2 a$, which is close to $\lambda=2.5 a$ as used in our simulations.

${ }^{11}$ V. A. Shklovskii, A. K. Soroka, and A. A. Soroka, JETP 89, 1138 (1999).

${ }^{12}$ R. Wördenweber, P. Dymashevski, and V. R. Misko, Phys. Rev. B 69, 184504 (2004).

${ }^{13}$ A. Pruymboom, P. H. Kes, E. van der Drift, and S. Radelaar, Phys. Rev. Lett. 60, 1430 (1988)
${ }^{14}$ N. Kokubo, R. Besseling, V. M. Vinokur, and P. H. Kes, Phys. Rev. Lett. 88, 247004 (2002).

${ }^{15}$ J. E. Villegas, S. Savel'ev, F. Nori, E. M. Gonzalez, J. V. Anguita, R. Garcia, and J. L. Vicent, Science 302, 1188 (2003).

${ }^{16}$ J. Van de Vondel, C. C. de Souza Silva, B. Y. Zhu, M. Morelle, and V. V. Moshchalkov, Phys. Rev. Lett. 94, 057003 (2005).

${ }^{17}$ A. V. Silhanek, J. Van de Vondel, V. V. Moshchalkov, A. Leo, V. Metlushko, B. Ilic, V. R. Misko, and F. M. Peeters, Appl. Phys. Lett. 92, 176101 (2008).

${ }^{18}$ G. D’Anna, V. Berseth, L. Forro, A. Erb, and E. Walker, Phys. Rev. B 61, 4215 (2000).

${ }^{19}$ H. Pastoriza, S. Candia, and G. Nieva, Phys. Rev. Lett. 83, 1026 (1999). 